\title{
Ueber die Endigungsweise der sensiblen Nerven.
}

Nach Untersuchungen von Dr. V. Izquierdo mitgetheilt

von

Professor Waldeyer.

Seit der Monographie W. Krause's über die terminalen Körperchen der einfach sensiblen Nerven und der Darstellung desselben Autors in seinem Lehrbuche der ,allgemeinen Anatomie" ist eine zusammenhängende und umfassende Bearbeitung aller Endigungsweisen sensibler Nerven nicht melr versucht worden; dagegen hat sich die Zahl der Detailbeobachtungen fast ins Unabsehbare aufgehäuft; die Referate über das in der Anatomie des Nervensystems Geleistete nehmen Jahr für Jahr den breitesten Platz in den Jahresberichten ein und unter den hier einschlägigen Untersuchungen finden sich stets die über Nervenendigungen in ansehn. licher Zahl.

Im verflossenen Jahre veranlasste ich einen meiner fähigsten Laboranten, Herrn Dr. V. Iz quierdo aus Santiajo (Chile), sich in dieses Capitel hineinzuarbeiten, um, womöglich, über einen oder den anderen Punct der noch zahlreich hier bestehenden Differenzen Entscheid und Auflklärung zu erzielen. Dr. Izquier do hat seine Untersuchungen ausgedehnt auf die Endigung der Nerven in der Hornhaut, in den Grandry'sehen Körperchen, in den sog. Endkolben der Genitalien und in den Pacinischen Körperchen. Diese Auswahl geschah unter Berücksichtigung der Thatsache, dass wir bei den sensiblen Nervenendigungen es vornehmlich mit zwei verschiedenen Endigungsformen zu thun haben. So weit wir bis jetzt wissen, endigen nämlich die Nerven entweder einfach in oder $\mathrm{z}$ wischen den sonstigen Gewebselementen, oder sie enden mittelst 
besonderer Vorrichtungen, die wir nach W. Krause's Vorgang als "Terminalkörperchen" bezeichnen. Wir werden später sehen, dass diese Eintheilung zweckmässiger durch eine den physiologischen und auch morphologischen Prinoipien mehr entsprechende $z u$ ersetzen ist.

Die Hornhaut wurde als der günstigste Boden für die erstere einfache Form der Endignng g’ewählt; für die zweite Form schien es erforderlich, um zu Vergleichungen gelangen zu könuen, mehrere Arten der bis jetzt beschriebenen Terminalkörperchen der Untersuchung zu unterwerfen.

Um bei der grossen Zahl der vorhandenen Detailangaben die leitenden Gesichtspuncte nicht $\mathrm{zu}$ verlieren und das allgemein anatouisch Wichtige festzuhalten, möchte ich zuvörderst, ehe ich die Resultate Izquierdo's mittheile, die vorbandenen Angaben über sensible Nervenendigungen kurz zusammenstellen und die sich ergebenden generellen Resultate hervorheben.

Nachdem friber fast nur die in der Haut sich verbreitenden Nerven als sensible angesehen wurden und bekannt waren, bat die neuere Zeit, insbesondere seit uns durch Max Sehultze in der Ueberosmiumsäure und durch Cohnheim in Goldchlorid zwei souverïne Nervenreagentien dargeboten wurden, begonnen, Nerven die man vor der Hand wohl nur als sensible deuten kann, fast in allen Organen und Geweben des Körpers aufzudecken. Leider ist es uns bis jetzt unmogglich in allen Fällen anatomisch die Diagnose zu stellen, ob eine aufgefundeue Nervenverzweignng bezw. Endigung eine sensible sei oder nicht. Steht doch selbst der Begriff eines "sensiblen" Nerven überhaupt nicht fest. Wir werden uns hier nach dem Herkommen richten und unter "sensiblen" Nerven alle diejenigen verstehen, deren Leistung in der UebermitteIung und Fortleitung von peripheren Zuständen oder Eindrücken besteht, welche nicht zu einer der sogenannten böheren Sinnesempfindung'sweisen: der Gesichts-, Gehörs-, Geruchs- und Geschmacksempfindung, fiihren. Positiv gefasst, fallen nach dieser Ausschliessung das ganze Heer der sog. Allgemeingefühle nebst den Tast- und Temperatur-Empfindungen den sensiblen Nerven anheim. Wir müssen alle diejenigen Nerven hierher rechnen, welche unserm Centralorgan Nachricht von den Zuständen der Spannung, Dehnung etc. der mechanischen Apparate sowie des Gefässsystems unseres Körpers geben, welche ferner die centripe- 
tale Verbindung zwischen den drüsigen Apparaten und dem centralen Nervensystem herstellen und in den grossen Reflexapparat des Organismus als ein Hauptfactor eingreifen. Wie man sieht, ein grosser Bereich, für.den es erst noch gilt die genaueren anatomischen und physiologischen Grenzen und Abtheilungen zu schaffen.

Nennen wir die Summe aller in Diensten dieser verschiedenen Functionen stehenden Nerven und Nervenenden ,sensibele", so sind ihnen physiologisch offenbar die "sensorischen" Nerven und Endigungen am nächsten verwandt; wir verstehen unter den letzteren bekanntlich die Nerven der vier höheren eben genannten Sinnesorgane, von denen drei sich scharf abgrenzen lassen, während das sensorische und sensible Gebiet des Glossopharyngeus nicht bestimmt auseinander zu halten ist.

Mit Sicherheit sind auch anatomisch die motorischen Nervenendigungen in den quergestreiften Muskeln von den sensiblen abzutrennen, seit man in neuerer Zeit besondere Nerven in den Muskeln und Sehnen kennen gelernt hat, welche kaum einer andern als einer centripetalen Leitung dienen können. Nicht so günstig steht es um die glatte Muskulatur, denn wir sind hier noch ausser Stande anatomisch die sensiblen von den motorischen Nervenenden zu scheiden.

Ganz unsicher ist ferner noch die anatomische Bestimmung: der Grenze der vierten Nervenabtheilung, der secretorischen und der vielumstrittenen fünften, der trophischen, gegen die sensibelen. Wenn wir einen Nerven in gewissen Gewebszellen oder in Drüsenzellen oder zwischen solchen sein Ende finden sehen, so ist es uns nach dem heutigen Stande unserer Kenntnisse unmöglich zu sagen, ob wir es mit einem secretorischen, trophischen oder sensibelen Nerven zu thun haben. Um also nicht in Willkürlichkeiten zu verfallen, müssen wir hier die anatomischen Grenzen noch weiter ziehen, als die physiologischen und zu dem hier zu besprechenden Gebiete noch alle diejenigen Nervenendigungen hinzuzählen, welche möglicherweise auch als trophische oder secretorische aufzufassen wären.

In diesem grossen Gebiete sind nun bislang von den verschiedenen Autoren nachstehende Nervenendigungsweisen thatsächlich beschrieben worden:

1) Freies Auslaufen der Nervenfäden in eine Spitze, oder Arohir f. mikrosk. Anatomie. Bd. 17. 
einfaches Auslaufen in ein Ende, wie das Ende eines abgeschnittenen Fadens. Dabei kann dieses Ende entweder frei uber das Niveau einer Körperfläche hinausragen, oder innerhalb der Gewebegrundsubstanz oder Kittsubstanz liegen, $z$ wischen den zelligen oder fasrigen Bestandtbeilen ohne mit einem dieser in Verbindung zu treten: Einfache freie Endigung.

Als Beispiele freier Enden markloser Nerven ohne Endknöpfchen seien hier nur erwähnt die von Morano (Arch. f. Ophth. von v. Graefe 17. Bd. Abth. II, 1872) beschriebenen Endigungen der Conjunctival-Nerven, und die Endigungen der Nerven in den Sehnen, wie sie neuerdings von te Gempt (Ein Beitrag zur Lehre von den Nervenendigungen im Bindegewebe, Dissert. inaug., Kiel, 1877) geschildert worden sind.

Freie Enden markhaltiger Nervenfasern will Engel in der Conjunctiva bulbi gesehen haben (Zcitschrift d. Gesellsch. d. Aerzte in Wien 1847 Heft 5.)

Offenbar sind aber auch hier noch mehrere Modificationen zu unterscheiden und mehrere Punkte bei der Constatirung solcher Nervenendigungen zu beachten. Zunächst kommt es darauf an, ob bei dieser Endigung der Nerv noch irgend eine Hülle besitzt, oder $o b$ er als blanker, nackter Axencylinder endigt, höchstens von der sog. Axencylinderscheide umgeben. Fernerhin ist als ein zu prüfender Umstand vielleicht nicht ohne Werth, ob das freie Ende einen ganzen Axencylinder repräsentirt, wie er als Axencylinder in einer hinteren Nervenwurzelfaser oder einer äquivalenten Hirnnervenfaser enthalten war, oder nur das Theilstick eines solchen, eine sogenannte „Axenfibrille". Die Frage ist zu beantworten, ob aberhaupt freie Endigungen ganzer sensibeler Axencylinder vorkommen?

2) Freies Auslaufen in ein Endknöpfehen (bouton terminal): Knopfförmige freie Endigung.

Hierbei kommen dieselben Modificationen in Betracht wie für Nro. 1.

Beispiele für diese Form der Nervenendigung sind in der Literatur reichlich verzeichnet. Hier sei nur an die bahnbrechenden Arbeiten von Hoyer und Cohnheim, s. Centralbl. f. d. medic. Wissensch. 186i, und Virchow's Arch. 38. Bd. 1867, erinnert, wo zum ersten Male diese Art der Nervenendigung im Epithel, und zwar im Epithel der Hornhaut, nachgewiesen wurde; die Enden sollten nach Cohnheim über das Niveau der Hornhaut hervorragen. Kölliker, Sitzgsber. der med. Ges. zu Würzburg, 30. Juni 1866, Hoyer, dieses Arch 9. Bd. p. 220, W. Krause, allgemeine Anatomie 1876, u. A. bestätigten im Wesentlichen diese Angaben für die Hornhaut, v. MIojsi sovics für die Haut- 
der Säugethiere, s. Wiener akad. Sitzungsber. 71. Bd. 1875. - Erwähnt sei hier noch, dass nach Bonnet, Morphol. Jabrb. IV. p. 329, in den Haarbälgen, welche mit Schwellkörpern versehen sind, die Nerven mit blasigen Endknöpfen endigen, während sie bei den übrigen, schwellkörperlosen Haaren an der Glashaut einfach frei auslaufen.

3) Endschlingen (Ansae terminales). Was darunter zu verstehen sei, ist allbekannt, so dass von einer genaneren Definition Umgang genommen werden kann.

Die Existenz von Endschlingen ist eine reine Hypothese, die durch kein anatomisches oderi physiologisches Faktum gut gestuitzt ist, wenngleich wir uns nicht verhehlen wollen, dass durch die Annahme der folgenden Categorie, der "terminalen Netze", die Endschlingen in einer modificirten Form wieder hergestellt werden.

4) Terminale Netze (Retia terminalia). Ich halte es nicht für überflüssig zu bemerken, dass man streng zwischen Netz und Plexus unterseheiden muss, und sehliesse ich mich der Definition Hoyers an, wie sie derselbe bei Gelegenheit seiner Untersuchungen über die Endigung der Cornealnerven 1. c. gegeben hat. Wir würden unter einem Plexus nur eine Durchflechtung: verschiedener Nervenfasern verstehen, ohne dass dieselben mit einander in eine anastomotisehe Verbindung treten, während bei der Netzbildung das Letztere der Fall ist. Demnach ist ein im strengen Wortsinne terminaler Plexus undenkbar, wohl aber ein derartiges Netz. Auch hierbei wäre wieder zu unterscheiden, ob die Elemente des Endnetzes nackten Axenfibrillen oder Axencylindern entsprechen, oder ob sie noch irgend eine Hülle besitzen.

Für die Existenz wirklich terminaler Netze treten ebenfalls zahlreiche Autoren ein. So J. Arnold (Die Bindehaut der Hornhaut und der Greisenbogen, Heidelberg 1860) in der Conjunctiva, Waldeyer (Graefe-Saemisch Handbuch der gesammten Augenheilk.) und E. Klein, (Quarterl. Journ. of mikr. Sc. Octob. 1871, p. 405) im Epithel der Cornea, neuerdings Jantschitz (Verhandl. der Russischen Naturforscher-Vers. für 1876 in Warschau) an verschiedenen Stellen der äusseren Haut, de Gempt, 1. c. im Peritoneum, Arnstein, (Sitzungsber. d. Wiener Akademie 1876. III. Abth. Octoberheft) für die äussere Haut der Mäuse u. A.

5) Endigung in oder mit einer Zelle. Bei dieser Form der Endigung, welche in einen gewissen Gegensatz mit den vorher aufgezählten Endigungsweisen tritt, lassen sich offenbar wieder mehrere Unterabtheilungen auseinanderbalten. Die Endigung kann zunächst stattfinden in einer Zelle, d. L., das Nerven- 
ende liegt im Innern einer Zelle, ohne aber mit der Zellsubstanz selbst zu verschmelzen. Die Zelle erscheint in einem solchen Falle mehr als eine Art Gehäuse für das im Inneren derselben deutlich als solches erkennbare Nervenende. So beschreibt es z. B. Letzerich (Virchow's Arch. 42. Bd. 1868) von den Nervenenden in den Samenkanälchen. Dann aber kann der Nerv mit einer Zelle endigen, d. h. mit der Zelle ve rschmel zen, derart, dass seine Substanz in die Zellsubstanz continuirlich und ohne Grenze ubergeht. In diesem Falle würde man die Zellen als terminale Ganglienzell e $\mathrm{n}$ bezeichnen können.

Diese Form der Nervenendigung ist in neuerer Zeit ganz besonders häufig beschrieben worden. Wenn wir von den Angaben Ditlevsens, s. u., Merkels, s. u. Langerhans', s. u. und Virchows Arch. 44 Bd. absehen, so ist besonders K ühne, (Untersuchungen über das Protoplasma und die Contractilität, Leipzig 1864) zu nennen, welcher die Nerven der Hornhaut in den Hornhautzellen in dieser Weise enden lässt. Auch Jantschitz, 1. c. behauptet dasselbe für die Nerven der Oberhaut.

Weiterbin können nun beide Endigungsweisen, wie sie eben fur das Zellprotoplasma angenommen wurden, auch für den Kern und für das Kernkörperchen gelten, wenn man auch für letzteres wohl nur von einer Endigung mit dem Nucleolus wird sprechen können. Zahlreiche Angaben liegen grade über die Endigungsweise sensibler Nerven im Kernkörperchen vor.

So unter Anderen von Hensen in den Kernkörperchen der Epithelzellen des Froschlarvenschwanzes (dies. Arch. IV. 1868 p. 111), von Lipmann für die Hornbautzellen und die Endothelzellen der membrana Descemetii (Virchow's Archiv 48 Bd. p. 218). Endigung im Kern (und auch im Kernkörperchen) von Hornhautzellen beschreibt Lavdowsky (dies. Archiv VIII. p. 538).

Endlich müssen wir noch nach dem Character der Zellen hier Unterabtheilungen machen: es können nämlich die Nerven sowohl in epithelialen und Driisenzellen, als in bindegewebigen und in Muskelzellen endigen, und wir dürfen gewiss auch eine ganze Klasse der hierhergehörigen Endzellen, wie vorhin schon bemerkt, als terminale Ganglienzellen bezeichnen.

Als Beispiele der Éndigung in Drüsenzellen möge an die bekannten Angaben Pflüger's, s. Artikel "Speicheldrüsen" in Stricker's Handbuch der Gewebelehre, erinnert werden.

Möge es nicht befremden, hier bei der Besprechung sensibeler Nervenenden, Endigungen in Muskelzellen aufgezählt zu finden, denn es ist sehr wohl möglich, dass ein Nerv, der sich mit einer 
Muskelzelle verbindet, kein motorischer, sondern ein sensibeler, centripetal leitender sei. Namentlich dürfte, wie bemerkt, bei den glatten Muskelfasern der Entscheid bier schwierig sein.

Ich will nicht verfehlen noch auf eine andere Schwierigkeit aufmerksam zu machen; die uns bei der sub Nr. 5 rubricirten Fndigungsform entgegentritt. Wir besitzen nämlich, wie vorhin bereits angedeutet, kein anatomisches Kriterium, um aussagen zu können, wenn wir einen Nerven cellulär endigen sehen, dieser Nerv sei ein sensibler, oder, um den Begriff weiter zu fassen, er leite centripetal. Er kann, da wir ja den Zellen Motilität als allgerneine Eigenschaft zusprechen, aueh centrifugal leiten, und, wenn wir von trophischen oder secretorischen Nerven sprechen, die jedenfalls hier in Frage kommen, so liegt für diese eine centrifugale Leitung ebenfalls vor.

6) Endigung in besonderen Apparaten. Seit Vater in Wittenberg und später Pacini, die gewöhnlich nach dem Letzteren benannten Körperchen entdeckt haben, ist die Aufmerksamkeit der Anatomen besonders auf bestimmte nervöse Endorgane gerichtet worden. Als solche müssen ausser den Pacini'schen Körperchen, die Meissner'schen Tastkörperchen, die Krause'schen Endkolben, die Genitalnervenkörperchen, Gelenknervenkörperchen und Endkapseln desselben Autors, sowie die Grandry'schen und Inzani'schen Körperchen, endlich die Sach s'schen, Rollett'schen und Golgi'schen Sehnenkörp e r cben genannt werden. Dass diese Schar von Nervenendkörperchen dem Gebiete der sensiblen Nervenendignngen zugerechnet werden müsse, darüber kann wohl kein Zweifel bestehen Die anatomische Untersuchung der in Rede stebenden Endorgane lässt tibrigens eine vereinfachte Eintheilung derselben zu und zwar in zwei Typen, wenn wir von den noch unsicheren Inzani'schen Körperchen absehen. - Der eine Typus ist repräsentirt darch die Pacini'schen Körperchen. Es gehören hierher noch die sogenannten cylindrischen Endkolben der Conjunctiva des Rindes, Schafes, Schweines, Pferdes a. s. w. und die „Endkapseln“, „Herbst'schen Kösperchen“ und "Tastkolben" W. Krause's, deren Bau in allem Wesentlichen dem der Pae in i'schen Körperchen gleichkommt. Dem zweiten Typus folgen die Grandry'schen Körperchen als einfachste Form und die Meissner'schen Tastkörperchen, vielleicht gehören auch die Endkolben der Conjunctiva des Menschen und der 
Affen, der Lippen, Zunge u. s.w., und die von W. Krause mit einem besonderen Namen ausgezeichneten Gelenk- und Genitalnervenkörperchen hierher. Indessen ist, wie wir weiter unten sehen werden, ein sicherer Entscheid darïber heute noch nicht möglich. Was die Inzani'schen Körperchen anlangt, so ist deren Existenz bislang noch eine zweifelhafte, so dass es gestattet sein mag, dieselben hier vorläufig unberiucksichtigt zu lassen.

Anders steht es mit den Sebnenendkörperchen, wie sie in neuester Zeit von Sachs, Rollett und Golgi beschrieben worden sind. Dieselben gehören unzweifelhaft zu den durch die Pacini'schen Körperchen repräsentirten Terminal-Apparaten, in denen die Nerven frei auslauten.

Der erste Typus, der der Pacini'schen Körperchen, den wir wohl am einfachsten in den von W. Krause sogenannten cylindrischen Endkolben mancher Säugethiere reprïsentirt finden, hat als charkteristische anatomische Eigenthümlichkeit die Endigung einer Nervenfaser innerhalb einer länglich-cylindrisch geformten granulirten Masse (Innenkolben) frei a usla ufend oder mit einer im Allgemeinen knopfförmig gestalteten Anschwellung. Der Innenkolben ist dann noch von diversen Hüllen in mehr oder weniger complicirter Weise umgeben und letztere Varietäten bedingen die verschiedenen Formen dieser Terminalkörperchen, die man geglanbt hat unterscheiden zu müssen.

Der zweite Typus, den wir am klarsten und einfachsten durch die Grandr y'schen Körperchen vertreten finden, kennzeichnet sich dadurch, dass der Nerv, gewöhnlich in mehrere Endäste getheilt, in dem Endkörperchen sich verbreitet; letzteres selbst besteht aus einer grösseren oder geringeren Anzahl von Zellen, die meist noch mit einer Hülle umgeben sind. Das Wesentliche ist nun, dass die Endäste der Nerven $\mathrm{z}$ wischen oder, wie Einige (Merkel für die Grandry'schen Körperchen, Waldeyer für die Endkolben der Conjunctiva) behaupten, in den genannten Zellen ihr Ende finden. Wo wir eine genauere Kenntniss dieses Endes haben, stellt es sich dar als eine platte Scheibe - Tastscheibe -, in welcher ein Nerven-Endast tibergeht und welche zwischen je zwei Zellen des Körperchens gelegen ist. Wenn wir annehmen, wozu ich mich wenigstens hinneige, dass die Tastscheibe einer modificirten Zelle entspricht, so wuirden wir in letz- 
ter Instanz in diesen Gebilden des zweiten Typus eine Endigung in Zellen wiederfinden. Merkel glaubt, die celluläre Endigung der Nerven hier in der Weise annehmen zu sollen, dass die Tastscheibe mit einer der benachbarten Zellen verschmelze.

Wir kommen später noch auf diese Frage zurick.

Kurz sei hier erwähnt, dass die von Inzani (Recherches sur la terminaison des nerfs dans les muqueuses des sinus frontaux et maxillaires, traduit par L. Jullien. Lyon médical, 18i2, Nr. 10) beschriebene Weise der Nervenendigungen in Folgendem besteht: Blasse aus wiederholter Theilung markhaltiger Nervenfasern hervorgegangene Fasern treten in birn- oder glockenförmige Kapseln ein, wo sie mit einer knopfförmigen Anschwellung versehen sind. Von dieser Anschwellung gehen aber wieder feinste Nervenfibrillen aus, welche die Kapsel verlassen und sich in dem umgebenden Gewebe verbreiten, um erst dort zwischen den diversen Gewebselementen mit ganz kieinen Knöpfchen zu endigen. Inzani, dem Jullien beistimmt, hält diese Endigungsweise in den Schleimhäuten, in den Nieren und im Peritoneum so wie an andern Lokalitäten für eine häufig vorkommende. Eine Bestätigung von anderer Seite haben diese Angaben bis jetzt nicht gefunden.

Eigenthümlich erscheinen, nach den bis jetzt darüber vorliegenden Angaben, auch tie Sehnennervenkörperchen, oder die "Nervenschollen" Rollett's zu sein. Nach der Darstellung des Letzteren zerfallen in den Sehnen markhaltige Nervenfasern büschelförmig in mehrere kurze Endzweige, welche, einfach zugespitzt, innerhalb einer kernhaltigen Substanz, der ${ }_{n}$ Endscholle“, die eincr motorischen Endplatte ähnlich ist, endigen. (Rollett in Wiener akad. Sitzungsber. III. Abth. Bd. 73 1876.) - Sachs, (s. Reicherts und Du Bois-Reymonds Arch. 1875), hatte äbnliche Bildungen ebenfalls beschrieben und fügt hinzu, dass die Sehnennerven mitunter auch in Endkolbenähnlichen Körperchen mit einer bläschenförmigen Anschwellung enden. Solche Endkolben in den Sehnen bestätigt auch neuerdings Golgi (Rendiconti del reale istit. lombardo, fasc. IX, p. 445, 18i8), fügt aber noch die Schilderung einer besonderen Endigungsweise hinzu, die in Folgendem besteht. Ungemein lange $(300-800 \mu)$ aus kernhaltigem, fikrillären Bindegewebe bestehende Körper erstrecken sich der Länge nach zwischen den Sehnenbündeln hin vom Muskelende beginnend. In diese Körper treten einige markhaltige Nervenfasern ein und verzweigen sich gegen besondere kleine Haufen körniger Substanz hin, welche an der Peripherie der grossen Körper gelegen sind; Verfasser meint, dass in diesen Haufen körniger Substanz die Nerven mit marklosen Terminalnetzen endigen.

Es hat nicht an Versuchen gefehlt, die zahlreichen bekannt gewordenen Nervenendigungsarten unter einzelne wenige Grundformen zu vertheilen, sie womöglich von einer einzigen Urform abzuleiten. W. Krause, in seinem inhaltreichen Handbuche der allgemeinen Anatomie, sieht in den cylindrischen Endkolben die 
Grundform für alle terminalen Körperchen, von der aus sich nach der einen Seite hin die Pacini'schen Körperchen mit ihren Verwandten, nach der anderen die Tastkörperchen, runden Endkolben u. s. w. entwickelt hätten. Mit dieser Anschauung sind die über die Tastkörperchen und Grandry'schen Körperchen seit Langerhans, Merkel, Ranvier, Hesse und Izqui erdo s. w. u. gewonnenen Erfahrungen unvereinbar, insofern die Endigungsweise der Nerven in beiderlei Endapparaten doch wesentlich differirt.

In noch mehr radicaler Weise versucht $D$ itlevsen neuerdings sämmtliche sensible Nerven unter einen Hut zu bringen. (Foelenervernes Endelse hos Mennesket og Hvirveldyret. Nord. med. Arkiv VIII, p. 11. Eingehendes Referat vom Verf. selbst in Virchow-Hirsch's Jahresbericht f. 1876. p. 61.) Er hält nämlich die Existenx freier Nervenenden für unerwiesen, sieht die terminalen Netze als irrthümlich an, und lässt als einzige Endigungsweise die cellulüre gelten. Was die Pacini'schen Körperchen anlangt, so meint er bei denen der Vögel z. Thl. einen zelligen Bau des Innenkolbens nachgewiesen zu haben, und wirft für die der Säugethiere die Frage auf, ob dieselben überhaupt als Endkörper von Gefïhlsnerven zu betrachten seien. Auch findet er die Oberhautnerven vom Frosch sämmtlich in Zellen iibergehen, welche dicht unter der oberen Hornschicht lagern.

Langerh an s (Zur Anatomie d. Amphioxus lanceolatus, dies. Arch. Bd. 12) hat sich bereits früher, wenn auch weniger bestimmt,. in ähnlicher Weise ausgesprochen. Ausgehend von der durch ihn festgestellten Thatsache, dass alle Hautnerven des Amphioxus in einfachen geisselfuhrenden Epithelzellen der Oberhaut enden (Fühlzellen Langerhans), dass man nirgends daselbst terminale Plexus oder Netze finde, scheint er diese Endigungsweise als die Grundform bei den Wirbelthieren anzusehen, von der sich auch die sogenannten freien Enden ableiten liessen, die er vermuthungsweise. wie z. B. in der Cornea, als reducirte celluläre Enden auffasst p. 306 .

Fr. Merkel, d. Arch. Bd. 11, nimmt wieder zwei Categorien von sensiblen Nervenenden (in der Haut) an, die celluläre, welche er für die den Tastnerven eigenthtimliche erklärt und die freie mit knopfförmigen Anschwellungen, letztere möchte er als Enden der Temperatar-Nerven ansehen.

Ich glaube nicht, dass man nach unseren heutigen Kenntnissen im Stande ist alle sensiblen, geschweige denn alle centripe- 
talleitenden Nervenenden auf eine einzige, und zwar eine celluläre Grundform zuriickzubringen. Man müsste etwa sonst ins Feld führen wollen, dass sich ja die peripheren Nervenfasern aus auswachsenden und mit einander verschmelzenden Zellen entwickelten. So weit ich sehe, kommen wir mit Merkel auf einen Dualismus hinaus, wenn wir den zur Zeit noch gut gestützten Angaben der Forscher Rechnung tragen und dürfen sogar dabei den Begriff der sensibeln Nerven so weit fassen, wie es hier geschehen ist. Doch möchte ich aber keineswegs dahin verstanden werden, als ob ich mit Merkel die freien Enden den Temperaturnerven, die cellulären den Tastnerven zuschriebe.

Ich möchte vielmehr in dieser Beziehung der Auffassung Grünhagens (s. dessen treffliche Darstellung in der von ihm besorgten neuesten Auflage des Funke'schen Lehrbuches der Physiologie) zustimmen, dass die cellulären Endigungen den Empfindungen des durch E. H. Weber besonders hervorgehobenen Druck- und Temperatursinnes dienen, welche Empfindungen sich bekanntlich stets mit der Vorstellung einer bestimmten objectiven ErregungsUrsache verknüpfen, während die frei endenden Nerven den sogenannten "Gemeingefiuhlen" zufallen. Welche Rolle dabei die besondern Terminalkörper spielen, lässt sich allerdings zur Zeit nicht präcisiren.

Hatten wir unter den Nervenendigungen ohne besondere Vorrichtungen solche, die wir als freie bezeichnen mussten und Endigung mit oder in Zellen, so kehren, wie wir gesehen baben, beide Endigungsformen auch bei den Terminalkörperchen wieder. Die $\mathrm{P}$ a cini'schen Körperchen repräsentiren den Typus der freie $\mathbf{n}$ Endigung, die Tastkörperchen die Endigung in Zellen, wenn wir uns der von mir hier ausgesprochnen Vermuthung anschliessen, dass die Tastscheibe eine modificirte Zelle sei. Somit könnten wir also die grosse Schaar der bisher beschriebenen sensibelen Nervenendigungen eintheilen in freie und celluläre. Jede dieser beiden Hauptendigungsweisen wäre nun entweder eine einfache oder mit einer besonderen Vorrichtung, einem terminalen Körperchen versehen, eine corpusculäre.

Der Durchfuhrung einer solchen einfachen Eintheilung stehen allerdings noch mehrere Umstände hindernd im Wege: das ist zunächst die Angabe vieler Forscher von der Existenz terminaler Netze, und dann die Unsicherheit, welche noch darüber besteht, 
ob wir in den disques tactiles Ranviers wirklich celluläre Enden erblicken dürfen. Ferner bleibt noch des Genaueren zu erforschen, wie sich die Nervenendigung in den Endkolben, Genitalnervenkörperchen und Gelenknervenkörperchen verhält. Die weitere Forschung hat besonders auf diese Punkte Gewicht zu legen, wobei nicht verschwiegen werden soll, dass auch noch eine grosse Anzahl anderer nicht minder wichtiger Fragen auf diesem Gebiete ihrer Lösung harren.

Einige dieser Fragen hat nun Dr. Izquierdo an der Hand der neueren Untersuchungsverfahren einer Entscheidung näher zu bringen gesucht und sind es haupsächlich folgende:

Gibt es in der That terminale Nervennetze? Welehes ist die Endigungsweise der Nerven in der einen Categorie der Terminalkörperchen, in den Tastkörperchen? welches die Endigungsweise in der zweiten Abtheilung, in den Pacini'schen Körperchen? Wie verhalten sich die Genitalnervenkörperchen zu den Pacini'schen Körperchen?

$\mathrm{Da}$ die terminalen Netze von den meisten Autoren, welche flir ihre Existenz eingetreten sind, in der Hornhaut aufgefunden worden sind, so war es natïrlich, auf die Untersuchung dieser Membran zu recurriren. Izquierdo bediente sich des neuerdings von Ranvier empfohlenen Verfahrens: Einlegen der frisch abgetragenen Hornhaut in frisch ausgepressten filtrirten Citronensaft für 5 Minuten, daun 20 Minuten in eine 1\% Goldchloridlösung (3 ccm. für jede Hornhaut), dann in $30 \mathrm{~g}$ mit zwei Tropfen Essigsïure angesäiluertes Wasser, woselbst die Cornea 3-4 Tage - bis zur vollständigen Reduction - verweilt. Man wartet zweckmässig so lange, bis die Hornhaut tief dunkel gervorden ist, um das Nachdunkeln der Schnitte zu vermeiden. Die Hornhaut kann dann in Alkohol gebracht und geschnitten werden. Die Hornhaut von Vögeln, besonders von Tauben und Kanarienvögeln, erwies sich als ein sehr geeignetes Object; schon v. Thanhoffer (Virchow's Arch. $63 \mathrm{Bd}$.) hat die Hornhaut der Vögel für diese Untersuchungen besonders empfohlen.

Die Nervenendigungsweise ist nun eine doppelte: entweder enden die feinsten nackten Axenfibrillen, in welche die Nerven schliesslich zerfallen, frei, oder im Protoplasma der Hornhautzellen. Niemals liess sich eine terminale Netzverbindung constatiren, weder innerhalb des Epithels noch im Hornhaut- 
stroma. In letzterem kommen, wenn auch sparsam, frei auslaufende Endigungen vor, die Mehrzahl der Stromanerven geht indessen in derselben Weise, wie es W. Kühne beschrieben hat, in das Protoplasma von Hornhautzellen über; dagegen war eine Endigung im Kern oder im Kernkörperchen nicht zu constatiren.

Innerhalb des Epithels gibt es nur frei e Nervenenden, entweder einfach auslanfend, oder mit einer kleinen Anschwellung versehen; es liess sich weder ein Netz noch ein Uebergang in Zellen constatiren.

Da ich noch vor wenigen Jahren die Existenz eines intraepithelialen Netzes vertreten, dagegen eine celluläre Endigung im Hornhautstroma geleugnet habe (1. c. Handb. der ges. Augenheilkunde v. Graefe und Saemisch), so musste es für mich besonders daran liegen, mich von der Richtigkeit der Ansichten Izquierdo's zu überzeugen. Ich kann nicht umbin, dieselben anzuerkennen. Nicht nur habe ich mich von der Verbindung der Nervenfäden mit dem Protoplasma der Hornhautzellen uiberzeugt, sondern ich glaube auch die Meinung von der Existenz terminaler Netze aufgeben zu müssen, da ich an den tadellosen Präparaten Izquierdo's Nichts dergleichen zu erkennen vermochte. Der Grund, weshalb ich mich früher für solohe Netze aussprach, ist vielleicht darin $\mathrm{zu}$ suchen, dass ich vor Allem menschliche Hornhäute untersuchte, in denen, wie es scheint, sich leicht die Kittsubstanz zwischen den Epithelzellen bei der Goldbehandlung färbt, und dadurch der Anschein von Anastomosen erzeugt wird.

Bezüglich der Nervenenden in den Tastkörperchen wandten wir uns zu den einfachen Formen derselben, als welche nach Merkels schönen Untersuchungen die Grandry'schen Körperchen gelten müssen; was für diese gilt, dürfen wir auf Grund der neueren Angaben Langerhans' (dies. Arch. IX) und Ranviers (Compt. rend. 1877. F. 85) auch unbedenklich auf die menschlichen Tastkörperchen übertragen. Untersucht wurden besonders die Tastkugeln (Hes se, Arch. v. His-Braune 1878) der Entenzunge mit 1/2 pr. Osmiumlösungen nach 24stündiger Einwirkung, später kamen die Präparate in $96 \%$ Alkohol, aus welchem sie geschnitten wurden. Auch 1/4 pr. Goldchloridlösung, in welcher die Stücke 1/2 Stunde verweilten, wurde verwendet. Izquierdo bestätigt nun den von Merkel (d. Arch. 1878) und Hesse 1. c. gefundenen 
„Plattenring“ als Theil der Kapsel und die zuerst von Ranvier als wirkliche Endigung hervorgehobene Nervenendigung in der Tastscheibe. Es sei mir gestattet hier zu bemerken, dass Izquierdo diese Endigung in der Tastscheibe völlig unabhängig von Ranvier aufgefunden hat, da uns des Letzteren Mittheilung in den Compt. rend. 26. Nov. 1877 erst später zu Händen kam. Uebrigens haben fast alle Autoren, welche vorher über die Grandry'schen Körperchen berichteten, diese Tastscheibe abgebildet, wenn sie dieselbe auch nicht für die Nervenendigung erklärten.

Izquierdo schildert nun die Tastscheiben als bestehend aus einer homogenen dunkleren Aussenschicht und einer inneren heller erscheinenden protoplasmatischen Masse; mitunter treten sie deutlich in bläschenförmiger Gestalt auf. Die Schwann'sche Scheide soll nun in die Aussenschicht, der Axencylinder in die protoplasmatische Binnenmasse der Tastscheibe iibergehen, eine continuirliche Verbindung der Tastscheibe mit den übrigen Zellen (Deckzellen) des kleinen Apparates, wie Merkel l. c. ihn constatirt haben will, konnte nicht nachgewiesen werden,

Izquierdo hat weiterhin anch die Entwi ckelung der Grandry'schen Körperchen untersucht und gefunden, dass dieselben als kleine Epithelzellenhäufchen entstehen, welche sich von der untersten Schicht des Rete malpighii in die betreffenden Papillenspitzen einsenken und spüter sich abschnüren; doch gelang es ihm nicht genaner den Zeitpunkt festzustellen, wann der Nerv mit den Körperchen in Verbindung tritt, wie das geschieht, und ob die Tastscheibe sich auch aus einer Zelle entwickelt. Nach meiner Einsicht der Präparate Izquierdo's möchte ich einen cellulären Ursprung der Tastscheiben annehmen und sie für modificirte Nervenendzellen erklären, womit auch ihr vorhin geschilderter feinen Bau übereinstimmt.

Die Pacini'schen Körperchen wurden besonders mit Rücksicht auf die Frage untersucht, ob innerhalb derselben der Nerv als solcher frei endet, sei es in eine Spitze auslaufend, oder in ein Endknöpfchen, oder ob das Ende des Nerven etwa noch innerhalb des Körperchens mit terminalen Zellen in Verbindung tritt.

Die Pacinischen Körperchen der Katze, welche vorzugsweise untersuchte, wurden, liessen nun ausnahmlos erkennen, dass der Nerv an seinem Ende nicht mit der Substanz des Innenkolbens oder der 
Kapsel in Verbindung tritt, sondern meist in eine verschieden gestaltete Verdickung ausgeht, die wie eine Anschwellung des fibrillär gebanten Axeneylinders selbst erscheint. Die mannichfachen Formen dieser „Endknöpfchen" oder „Endknospen“ sind von Axel Key und Retzius treffend beschrieben worden. (Studien in der Anatomie des Nervensystems und des Bindegewebes II. Stockholm. 1876.) In selteneren Fällen läuft der Axenfaden auch einfach in eine Spitze aus, was Axel Key und Retzi us nicht gesehen haben wollen. Ich kann nach zahlreichen eigenen Untersuchungen die Angaben Izquierdo's bestätigen.

Endlich wurden auch die Terminalkörperchen der Clitoris des Kaninchens untersucht, in denen stets ein einfaches Auslaufen des Nerven in eine Endspitze beobachtet wurde. Izqu ie rd o bezeichnet sie als Endkolben; ihre Gestalt ist, wie belsannt, eine sehr wechselnde, zwischen einer rundlichen und eylindrischen Form. Alle bestehen aus einer Kapsel, einem Innenkolben und dem in diesen eintretenden Nerven. Bemerkenswerth erscheint das Verhalten des Innenkolben, der zwar in der Mehrzahl der Fälle aus einer feingranulirten Masse besteht, in andern aber Kerne enthält und in wieder andern sich aus deutlich getrennten Zellen zusammengesetzt erweist. Man kann demnach Izquierdo wohl zustimmen, wenn er meint, dass die Innenkolben aus Zellen sich entwickeln, deren Protoplasma unter Schwund der Kerne verschmilat. Was aber das. Wesentliche ist, mochte die Structur des Innenkolbens eine noch deutlich zellige sein, oder nicht, stets war die Endigung des Nerven eine freie, niemals konnte man ihn mit Gebilden, wie etwa die Tastscheiben sie darstellen, oder gar mit Zellen verschmolzen sehen. Es müssen demnach diese Körperchen den $\mathrm{Pa}$ cinischen an die Seite gestellt werden.

Für mich lag es nahe, auch bei den Endkolben der Conjunctiva, für welche ich (dieses Arch. Bd. 11) eine zellige Structur des Innenkolbens und eine Verbindung des Nerven mit den Zellen behauptet habe, nach diesen neuen Erfahrungen wiederum nachzusehen. Leider war das Material, welches mir neuerdings zu Gebote stand, nicht hinreichend frisch, um zu einem Entscheid zu gelangen. Wird auch die zellige Structur der Biunenmasse der conjunctivalen Endkolben durch den gleichen Bau der Tastkörperchen und die Befunde Izquierdo's am Innenkolben der Genitalkörperchen gut gestützt, so fragt es sich dennoch, nachdem bei den 
ersteren die Tastscheiben, bei den letzteren durchweg freie Endigungen gefunden wurden, ob die von mir angenommene celluläre Nervenendigung die richtige ist. Wie bemerkt, kunnte ich bei wenig giinstigem Material für diesmal die Sache nicht zum Austrag bringen. 